\title{
Transcriptional regulation of HIV-1 host factor COMMD1 by the Sp family
}

\author{
ERIKO KUDO $^{1}$, MANABU TAURA ${ }^{1}$, MARY ANN SUICO $^{2}$, HIROKI GOTO ${ }^{1}$, \\ HIROFUMI KAI $^{2}$ and SEIJI OKADA ${ }^{1}$ \\ ${ }^{1}$ Division of Hematopoiesis, Center for AIDS Research, Kumamoto University, Kumamoto 860-0811; \\ ${ }^{2}$ Department of Molecular Medicine, Graduate School of Pharmaceutical Sciences, \\ Kumamoto University, Kumamoto 862-0973, Japan \\ Received July 6, 2017; Accepted January 9, 2018
}

DOI: $10.3892 / \mathrm{ijmm} .2018 .3386$

\begin{abstract}
Copper metabolism Murr1 domain containing 1 (COMMD1) has multiple functions in the regulation of protein stability at the plasma membrane and in the cytoplasm. However, the regulation of COMMD1 transcriptional has remained to be elucidated. In the present study, the 5'-flanking region $(-1,192 /+83 \mathrm{bp})$ of the human COMMD1 gene was cloned. It was observed that the COMMD1 promoter region contains GC-rich region that has 7 putative Sp1-binding sites via in silico analysis. The proximal promoter region at $-289 /+83$ bp was required for COMMD1 basal promoter activity by deletion constructs of COMMD1 promoter. Moreover, Sp1 inhibitor, mithramycin A, suppressed basal COMMD1 promoter activity. The Sp1-binding site (-11/-1 bp) in the proximal promoter region was a critical site for COMMD1 gene regulation by $\mathrm{Sp} 1$ and $\mathrm{Sp} 3$. Sp1 upregulated COMMD1 promoter activity, whereas Sp3 suppressed it. Endogenous Sp1 and $\mathrm{Sp} 3$ bound to the proximal promoter region of COMMD1. Taken together, $\mathrm{Spl}$ constitutively regulates the basal expression of the COMMD1 gene in human epithelial cell lines.
\end{abstract}

\section{Introduction}

Copper metabolism Murr1 domain containing 1 (COMMD1) is a member of the COMMD family, which has ten members. This protein family is distinguished by a unique C-terminal motif called the COMM domain $(1,2)$. COMMD1 was previously reported to regulate copper homeostasis by binding to ATPase copper transporting alpha/beta (ATP7A/B), which are associated with the copper storage disorders Menkes disease and Wilson's disease $(3,4)$. COMMD1 regulates the folding, stability, ubiquitination

Correspondence to: Professor Seiji Okada, Division of Hematopoiesis, Center for AIDS Research, Kumamoto University, 2-2-1 Honjo, Chuo-ku, Kumamoto 860-0811, Japan

E-mail: okadas@kumamoto-u.ac.jp

Key words: copper metabolism Murr1 domain containing 1, transcriptional regulation, $\mathrm{Sp} 1$ and protein degradation of its interaction partner proteins, including ATP7A, ATP7B and nuclear factor (NF)- $\mathrm{B}(5-8)$. COMMD1 has been reported as a human immunodeficiency virus (HIV)-1 host factor and inhibits HIV-1 replication by blocking the degradation of the inhibitor of $\mathrm{NF}-\kappa \mathrm{B}$ $(\mathrm{I} \kappa \mathrm{B}-\alpha)(9)$. A previous study by our group reported that I $\kappa$ B $-\alpha$ expression is increased by HIV-1 latent infection through the induction of COMMD1 expression. Induction of COMMD1 in HIV-1 latent-infected cells also maintains latent HIV-1 infection. The expression of the COMMD1 protein and mRNA in HIV-1 latent-infected myeloid cells is stronger than in parental cells (10). However, it remains elusive how COMMD1 transcription and expression are regulated. To the best of our knowledge, no previous studies have assessed COMMD1 transcriptional regulation.

The transcription factors specificity protein 1 (Sp1) and Sp3 belong to the $\mathrm{Sp}$ family, which has four members. Sp factors bind to GC-rich DNA sequences in human gene promoters. The Sp family is ubiquitously expressed and regulates a number of housekeeping and tissues-specific genes $(11,12)$. Sp1 and Sp3 have 90\% DNA sequence homology in the zinc finger-binding domain and exhibit similar specificities and affinities for DNA binding (13). However, although there are structural similarities between them, a functional comparison identified $\mathrm{Sp} 1$ as a transcriptional activator, with $\mathrm{Sp} 3$ being either a gene expression activator or repressor depending on the promoter structure or cell type $(13,14)$.

The present study hypothesized that basal COMMD1 transcription is regulated by $\mathrm{Sp} 1$ because COMMD1 expression is ubiquitous (1), and due to prospective consensus Sp binding sites identified in the human COMMD1 promoter region (Fig. 1A). In the present study, the $-1,192 /+83$ bp region of the COMMD1 promoter was cloned and the effects of Sp1 and Sp3 on the promoter activity and COMMD1 mRNA and protein expression were assessed with a luciferase assay using COMMD1 promoter constructs. It was demonstrated that Sp1 upregulates COMMD1 promoter activity as a transcriptional activator, while Sp3 suppresses COMMD1 promoter activity as a repressor. Sp1 regulates COMMD1 promoter activity via $-11 /-1$ bp of the Sp1-binding site and is required for the basal expression of COMMD1 in human cell lines. 


\section{Materials and methods}

Reagents, antibodies and plasmids. Mithramycin A was purchased from Sigma-Aldrich (Merck KGaA, Darmstadt, Germany). BAY 11-7085 was obtained from Tokyo Chemical Industry (Tokyo, Japan). Pan-JAK, a Janus kinase (JAK) inhibitor, was from Santa Cruz Biotechnology, Inc. (Dallas, TX, USA). An antibody to COMMD1 (cat. no. ab58322) was from Abcam Japan (Tokyo, Japan). Anti-heat shock cognate $71 \mathrm{kDa}$ protein (Hsc70; cat. no. SPA-815) was from Enzo Life Sciences (Farmingdale, NY, USA). Anti-Sp1 (cat. no. sc-59) and Sp3 (cat. no. sc-644X) for the chromatin immunoprecipitation (ChIP) assay were from Santa Cruz Biotechnology, Inc. Anti-histone deacetylase 1 (HDAC1; cat. no. 607401) was from BioLegend (San Diego, CA, USA). Anti-Sp1 was from Active Motif (Carlsbad, CA, USA). Horseradish peroxidase (HRP)-conjugated anti-mouse (cat. no. 7076) and anti-rabbit (cat. no. 7074) antibodies were from cell signaling technology (Danvers, MA). The pGL4.11 plasmid was from Promega Corp. (Madison, WI, USA). The pERV2/Sp1 construct was kindly provided by Dr. G. Suske (Institute for Molecular Biology and Tumour Research, Philipps-University of Marburg, Marburg, Germany). pN3-Sp3FL (plasmid no. 24541) was obtained from Addgene Inc. (Cambridge, MA, USA).

Cloning of the COMMD1 genomic 5'region and construction of luciferase reporter gene plasmids. The region $1.2 \mathrm{~kb}$ upstream of the COMMD1 gene and part of its 5' untranslated region (UTR) were amplified with primers and Takara LA Taq polymerase with GC buffer (Takara Bio, Inc., Otsu, Japan) using genomic DNA prepared from U937 cells as a template. The polymerase chain reaction (PCR) product was cloned into the pCR2.1 TOPO vector using the TA cloning kit (cat. no. K4520-01; Invitrogen; Thermo Fisher Scientific, Inc., Waltham, MA, USA). The COMMD1 promoter-luciferase reporter plasmid was constructed by subcloning the COMMD1 promoter fragment $(-1,192 /+83)$ into the XhoI-KpnI restriction site of the pGL4.11 vector (Promega Corp.). Deletion mutants of the COMMD1 promoter were constructed by a PCR-based approach using the $-1,192 /+83$ construct as a template and inserting the amplified fragments into the pGL4.11 vector. Point mutant constructs were prepared using the Quick change II XL site-directed mutagenesis kit (cat no. 200521; Stratagene, La Jolla, CA, USA) according to the manufacturer's instructions. The sequences of primers used for mutagenesis are listed in Table I. All mutant plasmids were generated using the $-283 /+83$ construct in the pGL4.11 vector as a template. Restriction sites were introduced into mutations for selection, and the incorporation of the mutation was verified by restriction digestion and sequencing. All plasmids used in the present study were sequenced with an ABI 3130 Genetic analyzer (Applied Biosystems; Thermo Fisher Scientific, Inc.).

Cell culture and transfection. The human embryonic kidney cell line $293 \mathrm{~T}$ and the human cervical carcinoma cell line HeLa were obtained from the American Type Culture Collection (Manassas, VA, USA) were maintained in Dulbecco's modified Eagle's medium supplemented with $10 \%$ fetal bovine serum (Nichirei Biosciences, Inc., Tokyo, Japan) at $37^{\circ} \mathrm{C}$ in a humidified atmosphere of $5 \% \mathrm{CO}_{2}$. Human promyeloid U937 were obtained from the American Type Culture Collection. Cells were cultured in RPMI-1640 (Invitrogen; Thermo Fisher Scientific, Inc.) supplemented with $10 \%$ fetal bovine serum and antibiotics at $37^{\circ} \mathrm{C}$ in a humidified atmosphere containing $5 \%$ $\mathrm{CO}_{2}$. Transient transfection of plasmid DNA was performed using HilyMax reagent (Dojindo Laboratories, Kumamoto, Japan) following the manufacturer's recommended protocol as described previously (15). Sp1 small-interfering RNA (si-RNA) was purchased from Sigma Aldrich (Merck KGaA). The transfection of Sp1 si-RNA was performed using TransIT-TKO reagent (Mirus Bio LLC, Madison, WI, USA) as described previously (16). The sequence of siRNA targeting Sp1 (si-Sp1) was as follows: Forward, 5'-CUACUACUACCA CCAGCA ATT-3' and reverse, 5'-UUGCUGGUGGUAGUA GUAGTT-3'. The negative control siRNA (MISSION siRNA Universal Negative Control; Sigma-Aldrich, Tokyo, Japan) was also used (con-si).

Reporter gene assays. 293T cells seeded onto 24-well plates were transfected with $0.1 \mu \mathrm{g}$ of the wild-type or mutant COMMD1 promoter in pGL4.11, together with a control Renilla luciferase plasmid (Promega Corp.). The amount of co-transfected Sp1 or Sp3 expression vector was as indicated in the figures. Spl inhibitor mithramycin A was added $24 \mathrm{~h}$ prior to harvesting the cells. At $48 \mathrm{~h}$ after transfection, cells were assayed for luciferase activity using a Dual-Luciferase Reporter Assay system (Promega Corp.) as described previously (16).

Western blot analysis. To analyze COMMD1 and Hsc70 protein expression, immunoblotting was performed as described previously (17). 293T cells were transfected with Sp1 and incubated for $72 \mathrm{~h}$. The protein lysate was recovered by radioimmunoprecipitation assay buffer as we described previously (18). For western blot analysis of Sp1 and HDAC1, nuclear extracts were prepared from cells according to a previously published protocol (17). The concentration of each sample was determined using a bicinchoninic acid assay kit (cat no. 23225; Thermo Fisher Scientific, Inc.). Proteins (10 $\mu \mathrm{g})$ were fractionated by $10 \%$ SDS-PAGE for COMMD1, Hsc70 and HDAC1 or $7.5 \%$ SDS-PAGE for Sp1. Proteins were transferred onto a polyvinylidene difluoride membranes. After blocking, the membrane was probed with the appropriate anti-COMMD1 (cat. no. ab58322; 1:1,000), Hsc70 (cat. no. SPA-815, 1:1,000), Sp1 (cat. no. sc-59, 1:1,000) and HDAC1 (cat. no. 607401, $1: 1,000)$ antibodies for $2 \mathrm{~h}$ at room temperature. Secondary IgG-HRP antibodies [anti-rabbit-HRP (cat. no. 7074) or mouse-HRP (cat. no. 7076); both 1:2,000] were added and incubated for $1 \mathrm{~h}$ at room temperature. The blot was visualized with Chemi-Lumi One Super (Nacalai Tesque, Kyoto, Japan). Band intensity was quantified with Image Gauge software (ver. 4.2; Fuji Film, Tokyo, Japan).

Reverse transcription-quantitative $(R T-q) P C R$. Total RNA was isolated from cells with RNAiso Plus (Takara Bio, Inc.) and cDNA was synthesized using the PrimeScript RT regent kit (Takara Bio, Inc.) according to the manufacturer's protocol. RT reaction was performed using the Takara PCR Thermal Cycler Dice (Takara Bio, Inc.) with the following conditions: $37^{\circ} \mathrm{C}$ for $15 \mathrm{~min}, 85^{\circ} \mathrm{C}$ for $5 \mathrm{sec}$. Real-time PCR amplification of COMMD1, Sp1 and internal control 18S ribosomal 
Table I. Primers for COMMD1 promoter cloning and mutagenesis of specificity protein 1-binding sites.

\begin{tabular}{ll}
\hline Primer name & \multicolumn{1}{c}{ Sequence (5'-3') } \\
\hline 5' COMMD1 prom (-1192_KpnI) & GGTACCCTGCCCTCCCTCTGGAGTCACCAATAC \\
5' COMMD1prom (-519_KpnI) & GGTACCGAGCCACTGATCTAGCTGGAAAGAC \\
5' COMMD1prom (-283_KpnI) & GGTACCTCTCCAGTAATCTCTGCGAGCAATC \\
5' COMMD1prom (-29_KpnI) & GGTACCGGCACGGCTCAGCTGTTGCGGGGC \\
3' COMMD1prom (+83_XhoI) & CTCGAGGAAAGTGTCCTGGGCCAGCGCATTC \\
COMMD1prom mt1 Forward & CCGTGGAGGTATTCTAAGGTCATTGGTGTTGCAGAGCAAAGCT \\
COMMD1prom mt1 Reverse & AGCTTTGCTCTGCAACACCAATGACCTTAGAATACCTCCACGG \\
COMMD1 prom mt2 Forward & GCACATCTCGGCCGCCGTATACCGGCACGGCTCAGCTG \\
COMMD1prom mt2 Reverse & CAGCTGAGCCGTGCCGGTATACGGCGGCCGAGATGTGC \\
COMMD1 prom mt3 Forward & CGGCTCAGCTGTTGCGGCCATCGGCCTTCGCAGAGCATG \\
COMMD1prom mt3 Reverse & CATGCTCTGCGAAGGCCGATGGCCGCAACAGCTGAGCCG \\
COMMD1prom mt2-2 Forward & GCACATCTCGGCCGCCGTCCATCGGCACGGCTCAGCTG \\
COMMD1 prom mt2-2 Reverse & CAGCTGAGCCGTGCCGATGGACGGCGGCCGAGATGTGC \\
\hline
\end{tabular}

COMMD1, copper metabolism Murr1 domain containing 1; prom, promoter; mt, mutant.

Table II. Primers used for the reverse transcription-quantitative polymerase chain reaction analysis.

\begin{tabular}{lll}
\hline Gene & \multicolumn{1}{c}{ Forward (5'-3') } & \multicolumn{1}{c}{ Reverse (5'-3') } \\
\hline COMMD1 & CTGGAGGCATTCTTGACTGCTC & GCTCTCACGGATTTTTGTCTTGTG \\
Sp1 & TCACTGTGAATGCTGCTCAACTCTC & AGACCAAGCTGAGCTCCATGATCAC \\
Sp3 & CTGTCCCAATGTAAAGAAGGTG & AGAATGCCAACGCAGATGAG \\
rRNA 18s & CGGCTACCACATCCAAGGAA & GCTGGAATTACCGCGGCT \\
\hline
\end{tabular}

COMMD1, copper metabolism Murr1 domain containing 1; Sp, specificity protein; rRNA 18s, 18 s ribosomal RNA.

(r)RNA were performed using the Fast SYBR-Green Master Mix (Applied Biosystems; Thermo Fisher Scientific, Inc.) following the manufacturer's instructions and as described previously (10). PCR amplifications were performed using the StepOne real-time PCR system with the following amplification conditions: $95^{\circ} \mathrm{C}$ for $3 \mathrm{~min}, 40$ cycles at $95^{\circ} \mathrm{C}$ for $10 \mathrm{sec}$, at $55^{\circ} \mathrm{C}$ for $30 \mathrm{sec}$. The $\mathrm{C}_{\mathrm{q}}$ values for each gene amplification were normalized by subtracting the $\mathrm{C}_{\mathrm{q}}$ value calculated for $18 \mathrm{~S}$ rRNA. Normalized gene expression values were presented as the relative quantity of each gene-specific mRNA according to $2^{-\Delta \Delta C q}$ method (19). The oligonucleotide primers used for real-time qPCR amplifications are listed in Table II.

ChIP assay. To examine the binding of Sp1 to the COMMD1 promoter, the nuclear extract of $293 \mathrm{~T}$ cells was used for the chromatin immunoprecipitation (ChIP) assay according to a previously described method (16). Cells were cross-linked using formaldehyde ( $1 \%$ final concentration) added directly to the cell culture media at $37^{\circ} \mathrm{C}$ for $15 \mathrm{~min}$, and the reaction was stopped by adding glycine ( $0.125 \mathrm{M}$ final concentration). Cells were rinsed with cold PBS and resuspended in cell lysis buffer. This mixture was incubated on ice for $10 \mathrm{~min}$ and then homogenized. The nuclei were resuspended in nucleus lysis buffer and incubated on ice for $10 \mathrm{~min}$. the samples were sonicated on ice with the ultrasonic homogenizer VP-050 (TAITEC, Saitama, Japan). The chromatin solution was precleaned using Staphlococcus aureus protein A-positive cells (Pansorbin, \#507862; Merck KGaA). Two micrograms of anti-Sp1 or anti-Sp3 antibody was incubated with pre-cleared chromatin. PCR was performed using the Fast SYBR green master mix as above. The primer set for measuring Sp1 and Sp3 binding activities was as follows: $-0.1 \mathrm{~kb}$ sense, 5'-GGTACCTCTCCA GTAATCTCTGCGAGCAATC-3' and antisense, 5'-CTCGAG GAAAGTGTCCTGGGCCAGCGCATTC-3'.

Statistical analysis. Values are expressed as the mean \pm standard error. Data were analyzed by a one-way analysis of variance with the Tukey-Kramer multiple-comparisons test or Dunnett's test, or by Student's t-test. JMP software (ver. 8.0.2; SAS Institute, Cary, NC, USA) was used for all statistical analyses. $\mathrm{P}<0.05$ was considered to indicate a statistically significant difference.

\section{Results}

Cloning of the 5'-flanking region of COMMD1 and analysis of putative transcription factor binding sites. To characterize the COMMD1 transcriptional system, a genomic DNA fragment 


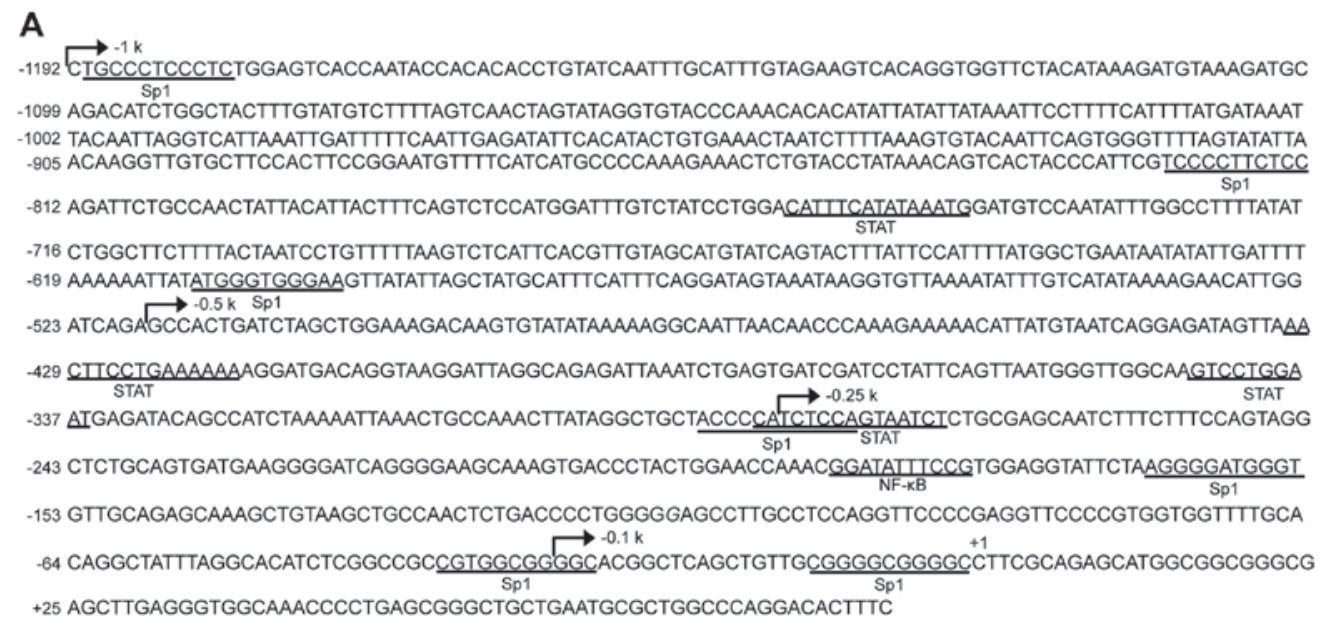

B

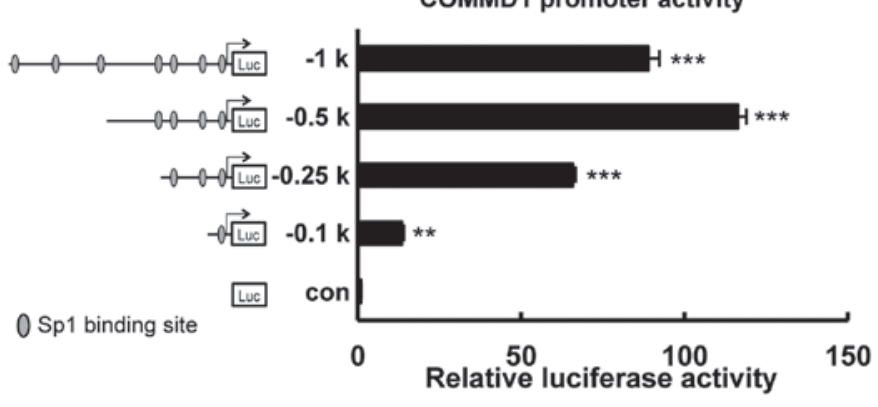

C

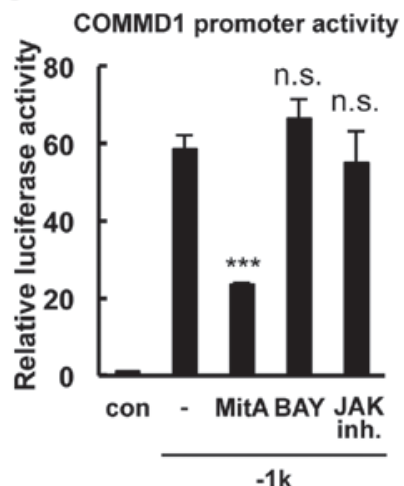

Figure 1. Identification of the minimal promoter region required for basal COMMD1 promoter activity. (A) Nucleotide sequence of the 5'flanking region of the human COMMD1 gene. The site indicated by $(+1)$ denotes the start site of transcription. The predicted binding sites for Sp1, STAT and NF- $\mathrm{BB}$ are marked on the sequence. The lengths of the different promoter constructs are indicated by arrows. (B) Deletion analysis of the COMMD1 promoter. 293T cells were transfected with COMMD1 promoter deletion constructs and lysates were harvested $48 \mathrm{~h}$ later for the luciferase reporter assay. Reporter activity is expressed as fold activation over the empty vector. (C) 293T cells were transfected with the COMMD1 promoter (-1 k) and treated with MitA, BAY or JAK inh. for $24 \mathrm{~h}$. Lysates were harvested $48 \mathrm{~h}$ later for the luciferase reporter assay. Values are expressed as the mean \pm standard error from three independent experiments. In (A) and (B), P-values were determined by analysis of variance followed by with Dunnett's test. ${ }^{* *} \mathrm{P}<0.01$ and ${ }^{* * * *} \mathrm{P}<0.001$, respectively, vs. the con or blank group. ns, not significant; SP, specificity protein; STAT, signal transducer and activator of transcription; NF, nuclear factor; Luc, luciferase; JAK inh., Janus kinase inhibitor Pan-JAK; con, control; COMMD1, copper metabolism Murrl domain containing 1; MitA, mithramycin A; BAY, BAY 11-7085.

containing the 5'-flanking region of the human COMMD1 gene (GenBank accession no. NC_000002.12) was cloned. The cloned fragment spans 1.2-kb upstream of exon 1, down the length of the first exon to 72 bp of the 5'UTR. An analysis of the COMMD1 5'-flanking region using the JASPAR database (http://jaspar.genereg.net) revealed a GC-rich region that has 7 putative binding sites for Spl within -1,192 bp upstream of the transcriptional starting site, which was designated as +1 (Fig. 1A). To identify the probable minimal promoter region required for the basal transcriptional activity of the COMMD1 gene, deletion constructs that contained 7, 4, 3 and $1 \mathrm{Sp} 1$ binding sites were generated (Fig. 1B). These constructs were transfected into $293 \mathrm{~T}$ cells that express COMMD1. The $-519 /+83$ bp construct had the highest activity among all constructs. The activities of the $-1,192 /+83,-519 /+83,-283 /+83$ and $-29 /+83$ bp constructs containing 7, 4, 3 and 1 Spl binding sites, respectively, were 88-, 116-, 65- and 13-fold higher than the basal activity, respectively. Putative binding sites for NF- $\kappa B$ and signal transducer and activator of transcription (STAT) transcription factors were detected in this region in an in silico analysis. However, blockage of the NF- $\kappa \mathrm{B}$,
STAT and JAK activation pathways did not affect COMMD1 promoter activity (Fig. 1C). Thus, these results indicated that the cis-regulatory elements required for COMMD1 transcriptional activity are mainly located in the core promoter region of -283 to $-1 \mathrm{bp}$.

Spl activates COMMD1 promoter activity. To clarify whether these Sp1 sites are involved in COMMD1 transcription, the $-0.25-\mathrm{kb}$ construct and increasing amounts of the Sp1-expressing plasmid were co-transfected into 293T cells. An increase in COMMD1 reporter activity was identified in 293T cells that was dependent on the amount of Sp1 expression vector (Fig. 2A), which was in parallel with the amount of Sp1 mRNA expression (Fig. 2B). The overexpression of Sp1 also increased COMMD1 mRNA and protein levels (Fig. 2C and D). These results suggested that Sp1 enhances the promoter activity of COMMD1.

Inhibition of Sp1 downregulates endogenous COMMDI expression. The present study analyzed the effects of mithramycin $\mathrm{A}$, a drug known to modify the GC-rich region of 


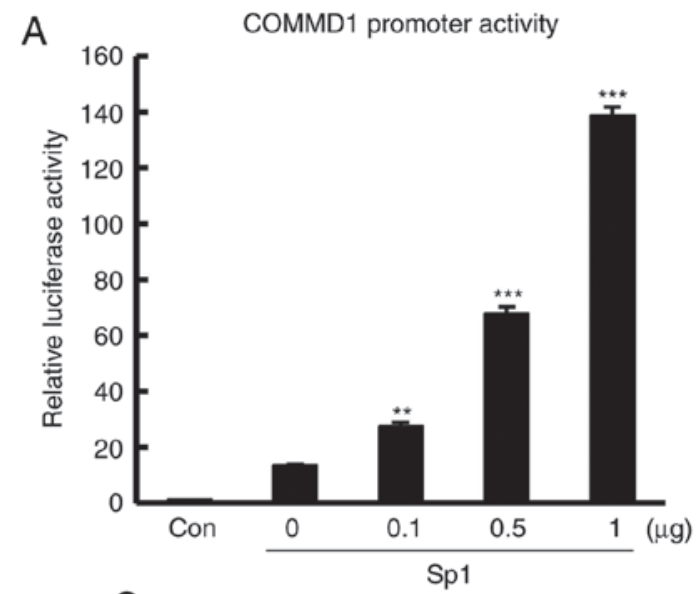

B
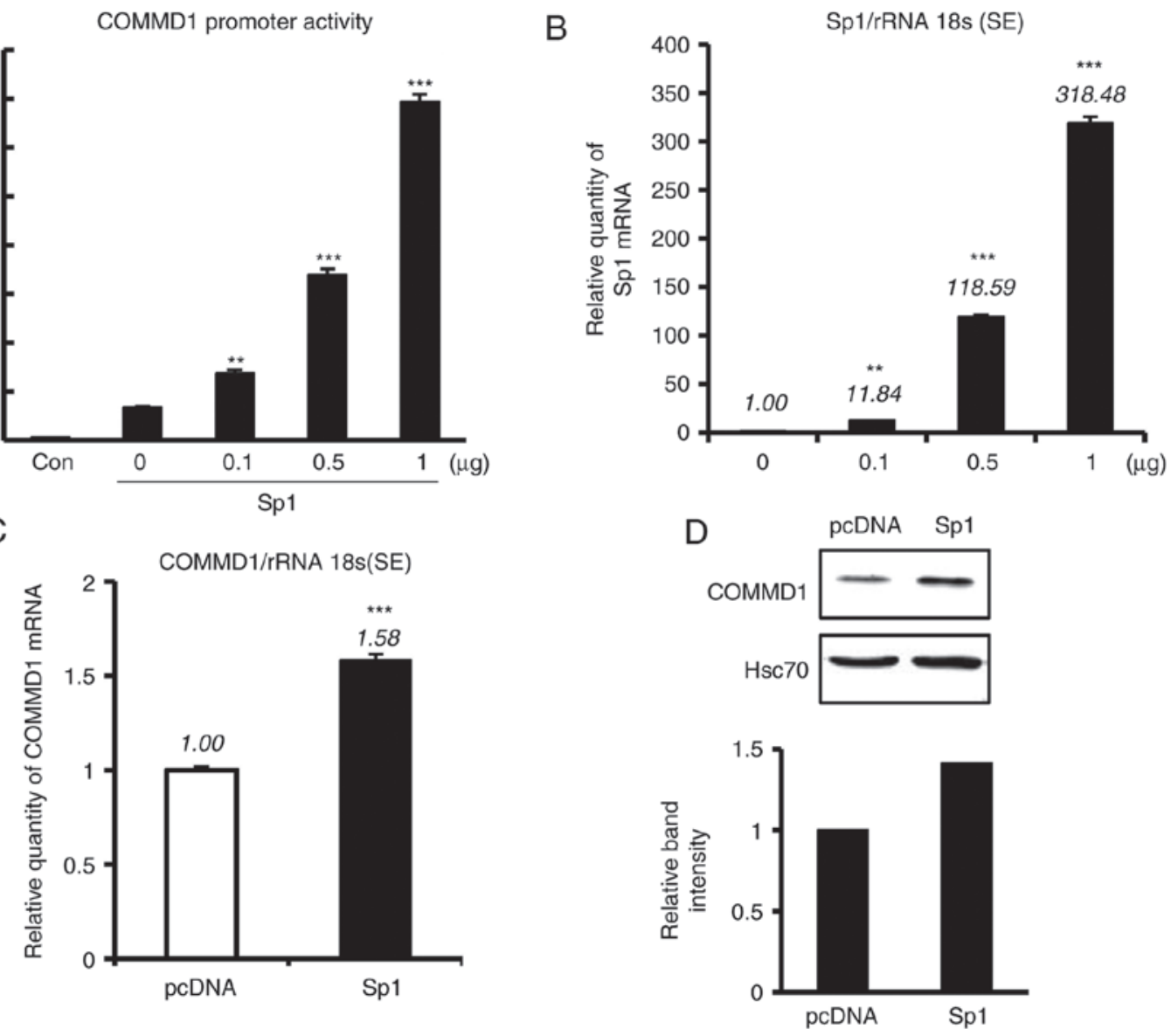

Figure 2. Sp1 upregulates COMMD1 promoter activity. (A) The COMMD1 promoter (-0.25 kb) was co-transfected with the indicated amount of Sp1 into 293T cells. Empty vectors were added to ensure a constant input of DNA. (B) Sp1 mRNA expression in 293T cells transfected with the Sp1 expression vector for $48 \mathrm{~h}$. Empty vectors were added to ensure a constant input of DNA. (C) 293T cells were transfected with the Sp1 expression vector, and mRNA was harvested for reverse transcription-quantitative polymerase chain reaction analysis $24 \mathrm{~h}$ later. (D) Protein expression in 293T cells transfected with the Spl expression vector for $72 \mathrm{~h}$. Band intensities were quantified and the relative ratios of the indicated proteins are displayed in a bar graph. Values are expressed as the mean \pm standard error from three independent experiments. P-values were determined by analysis of variance followed by the Tukey-Kramer test in (A) and (B) and by Student's t-test in $(C) .{ }^{* *} \mathrm{P}<0.01$ and ${ }^{* * *} \mathrm{P}<0.001$ vs. pcDNA. SP, specificity protein; COMMD1, copper metabolism Murrl domain containing 1; rRNA 18s, $18 \mathrm{~s}$ ribosomal RNA; con, control; HSC70, heat shock cognate $71 \mathrm{kDa}$ protein.

DNA and inhibit Spl binding (20). As presented in Fig. 3A, treatment with mithramycin A downregulated the promoter activity of COMMD1. The mRNA and protein expression of COMMD1 was also decreased with mithramycin A treatment (Fig. 3B and C). As mithramycin A has broad effects and induces cell death, the present study also applied Sp1-specific si-RNA, si-Sp1, which was transfected into HeLa cells (Fig. 3D). Mild Sp1 knockdown suppressed the mRNA and protein expression of COMMD1 (Fig. 3E and F). Collectively, these results suggest a role for Sp1 in regulating the COMMD1 promoter.

Sp3 suppresses the activity of the promoter of COMMD1, but not its expression. $\mathrm{Sp} 1$ and $\mathrm{Sp} 3$ have similar structures and bind to the same Spl-binding sites. However, their DNA-binding properties and regulatory functions differ depending on the promoter region or cellular background (14). The present study investigated whether Sp3 regulates COMMD1 promoter activity (Fig. 4). 293T cells were co-transfected with the $-0.25-\mathrm{kb}$ construct and increasing amounts of the $\mathrm{Sp} 3$ expression plasmid, and decrease in reporter activity was observed (Fig. 4A). In addition, to assess the association between Sp1 and Sp3 in the COMMD1 promoter region, the $-0.25-\mathrm{kb}$ construct was co-transfected with expression vector of Sp1 and Sp3 into 293T cells. Of note, COMMD1 promoter activity was upregulated by $\mathrm{Sp} 1$ and was suppressed by Sp3 (Fig. 4B). However, when Sp3 was transfected into 293 T cells (Fig. 4D), the expression of COMMD1 mRNA and protein was not suppressed (Fig. 4C and E). It was then investigated whether Sp3 decreases COMMD1 expression in Sp1-silenced cells. Sp1 knockdown decreased the expression of COMMD1 mRNA, and this decrease was enhanced by $\mathrm{Sp} 3$ overexpression (Fig. 4F). Thus, taken together, the results indicated that $\mathrm{Sp} 3$ regulates the transcription of COMMD1 in the absence of Sp1.

The -11/-1 bp Sp1-binding site is essential for COMMD1 promoter activity. Next, the present study investigated which Sp1 site is indispensable for the proximal promoter activity of COMMD1. Reporter constructs carrying a mutation in the Sp1-binding sites [mutant (mt)1, -11/-1; mt2, -37/-27; $\mathrm{mt} 3,-164 /-154]$ were created and their activities in 293T cells were analyzed (Fig. 5A and B). The mutation in the -164/-154 Sp1-binding site (mt3) had no significant effect on 
A

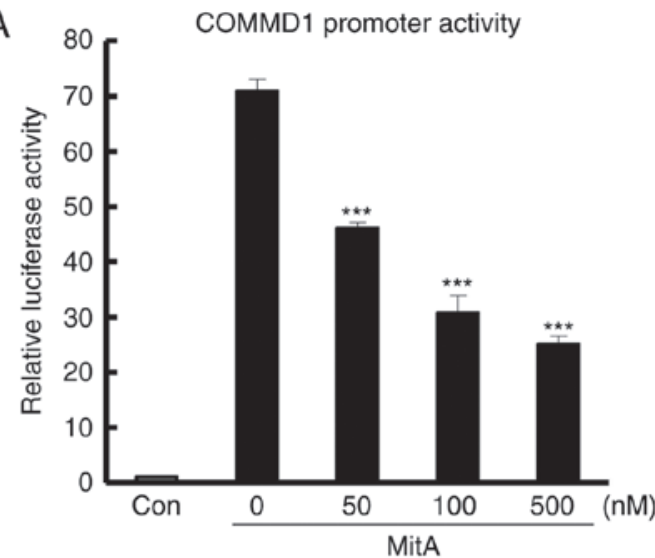

C
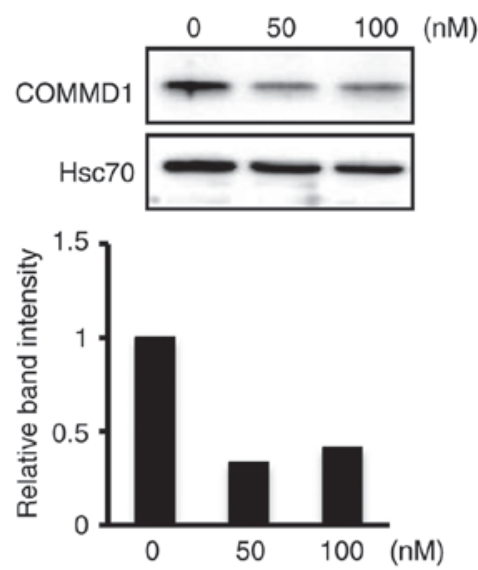

B

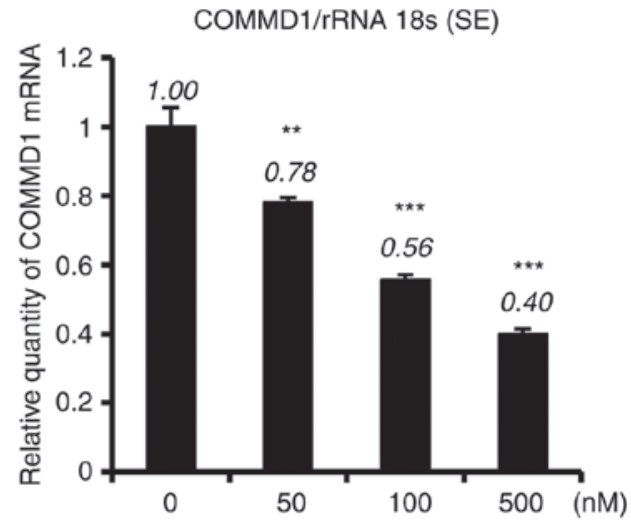

\section{E}

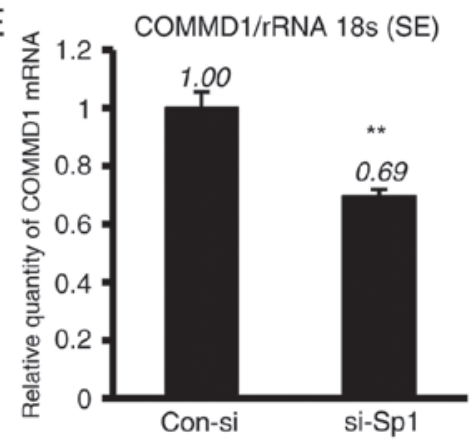

$\mathrm{F}$

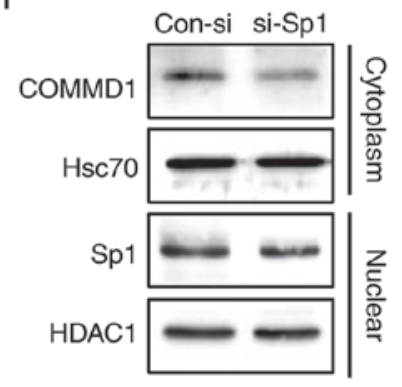

Sp1/rRNA 18s (SE)

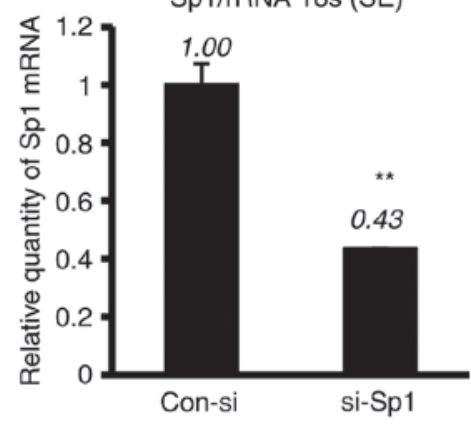

Figure 3. MitA and si-Sp1 suppress the expression and promoter activity of COMMD1. (A) 293T cells were transfected with the COMMD1 promoter (-0.25 kb) and treated with MitA for $24 \mathrm{~h}$ at the indicated concentration. (B and C) COMMD1 mRNA and protein expression in 293T cells treated with MitA for 24 and 72 h, respectively. (D) Sp1 mRNA expression in HeLa cells transfected with si-Sp1 or con-si. (E and F) COMMD1 mRNA and protein expression, respectively, in HeLa cells transfected with si-Spl or con-si. Band intensities were quantified and the relative ratios of the proteins indicated are presented in a bar graph. Values are expressed as the mean \pm standard error from three independent experiments. In (A) and (B), P-values were determined by analysis of variance followed by the Tukey-Kramer test and Dunnett's test, respectively. In E and F, P-values were assessed using Student's t-test. ${ }^{* *} \mathrm{P}<0.01$ and ${ }^{* * *} \mathrm{P}<0.001$, respectively, vs. pcDNA or the control. si-SP1, small interfering RNA targeting specificity protein 1; COMMD1, copper metabolism Murr1 domain containing 1; MitA, mithramycin A; rRNA 18s, 18 s ribosomal RNA; con, control; HSC70, heat shock cognate 71 kDa protein; HDAC1, histone deacetylase 1.

promoter activity. The mutation in the -11/-1 Sp1-binding site (mt1) completely abolished COMMD1 promoter activity. By contrast, the mutation in the $-37 /-27$ Sp1-binding site (mt2) led to a stronger COMMD1 promoter activity than that with the wild-type promoter. To confirm whether Sp1-binding site (mt2) led to a COMMD1 promoter activity, we created another mutant construct of different sequence of $\mathrm{mt} 2$ (mt2-2). Mt2-2 also led to a stronger COMMD1 promoter activity (Fig. 5B). The double mutant constructs containing $\mathrm{mt} 1(\mathrm{mt} 1+\mathrm{mt} 2$ and $\mathrm{mt} 1+\mathrm{mt} 3)$ abolished the promoter activity. These results indicated that $\mathrm{Sp1}$ regulates COMMD1 through the -11/-1 Sp1-binding site, which is crucial for the induction of COMMD1 promoter activity. To examine whether Sp1 or $\mathrm{Sp} 3$ binds to the promoter of COMMD1, a ChIP assay was performed using nuclear extracts from $293 \mathrm{~T}$ cells and anti-Sp1 or anti-Sp3 antibody. We observed that endogenous $\mathrm{Sp} 1$ or Sp3 binds to the promoter of COMMD1 as determined by ChIP assay (Fig. 5C). Taken together, these results indicate that Sp1 binds to the $-11 /-1$ bp Sp1-binding site of the COMMD1 proximal promoter region to activate COMMD1 promoter activity and is required for the basal expression of COMMD1. 

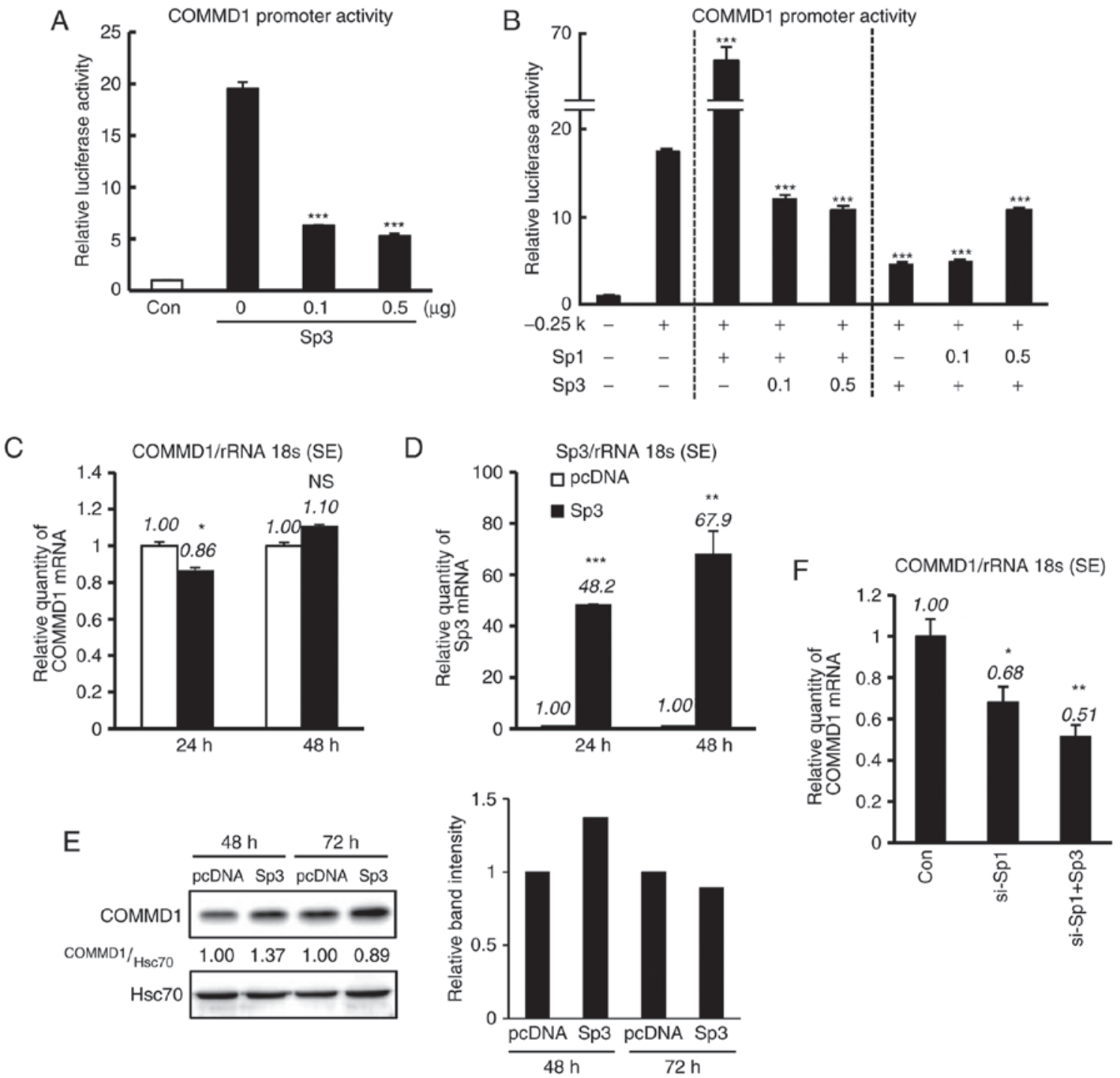

Figure 4. Sp3 downregulates COMMD1 promoter activity, but not its expression. (A) The COMMD1 promoter (-0.25 kb) was co-transfected with the indicated amount of Sp3 into 293T cells. Empty vectors were added to ensure a constant input of DNA. (B) The COMMD1 promoter (-0.25 kb) was co-transfected with the indicated amounts of Sp1 and Sp3 into 293T cells. Luciferase activity in lysates was measured $48 \mathrm{~h}$ after transfection. (C and D) COMMD1 and Sp3 mRNA expression in 293T cells transfected with pcDNA (white bar) or Sp3 (black bar). (E) COMMD1 protein expression in 293T cells transfected with pcDNA or Sp3. (F) si-Sp1 with or without Sp3 was co-transfected into HeLa cells for $24 \mathrm{~h}$. Band intensities were quantified and the relative ratios of the indicated proteins are displayed in bar graph. Values are expressed as the mean \pm standard error from three independent experiments. In (A), (B) and (F), P-values were determined by ANOVA followed by the Tukey-Kramer test. In (C) and (D), P-values were assessed using Student's t-test. ${ }^{*} \mathrm{P}<0.05,{ }^{* * *} \mathrm{P}<0.01$ and ${ }^{* * * *} \mathrm{P}<0.001$ vs. pcDNA, $-0.25 \mathrm{~kb}$ or the control. ns, not significant; COMMD1, copper metabolism Murrl domain containing 1; si-SP1, small interfering RNA targeting specificity protein 1; rRNA 18s, 18 s ribosomal RNA; con, control; HSC70, heat shock cognate $71 \mathrm{kDa}$ protein.

\section{Discussion}

COMMD1 was previously reported to interact with the copper ion channel ATP7A/B and is involved in copper transport in hepatocytes (2). COMMD1 expression is suggested to correlate with tumor malignancy, inflammation and anti-viral host defenses. In human cancers, including breast and prostate cancers, COMMD1 expression is frequently suppressed, which leads to increased tumor invasion in these patients (8). COMMD1 mRNA expression was demonstrated to be reduced in circulating leukocytes from inflammatory bowel disease (IBD) patients and the decrease in COMMD1 expression induced constitutive inflammation (21). A recent study indicated that microRNA-205 suppressed COMMD1 expression in stemness-enriched cancer cells (22). The present study was the first to report the cloning and characterization of the COMMD1 promoter region. The 5'-flanking region of COMMD1 is GC-rich and contains putative Sp1 consensus sites. COMMD1 is mainly regulated by the $\mathrm{Sp} 1$ transcription factor through the direct binding of Sp1 to the COMMD1 promoter region.

Sp1 upregulates a variety of genes, including house-keeping genes and tissue- or cell type-specific genes (23). An early study demonstrated that the expression of the human CD14 gene is upregulated by $\mathrm{Sp} 1$ in monocytic cells (24). Interleukin-10 expression is also regulated by $\mathrm{Sp} 1$ and $\mathrm{Sp} 3$ in a manner similar to that of other of house-keeping genes (25). The present study examined whether COMMD1 expression is regulated by Sp1. COMMD1 transcription and expression were identified to be upregulated by $\mathrm{Sp} 1$. Sp1 is essential for early embryonic devel- 
A

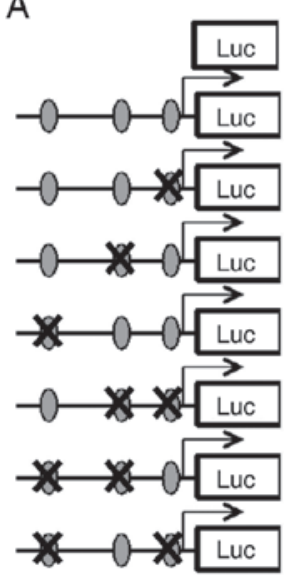

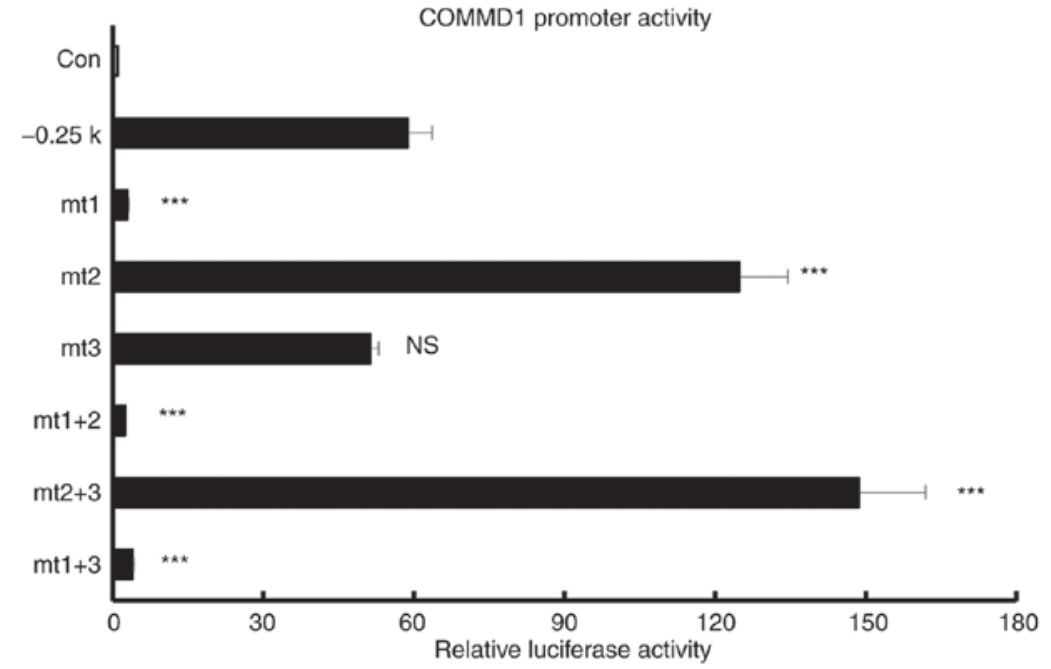

C

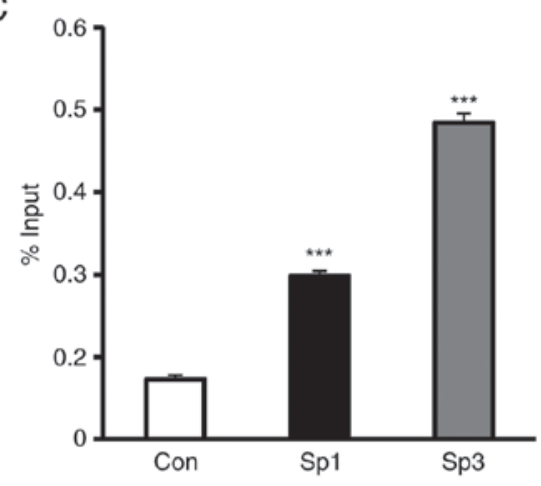

Figure 5. Sp1 regulates COMMD1 gene expression via direct binding to the -11/-1bp Sp1-binding site of the COMMD1 proximal promoter region. (A and B) A mutant plasmid was transfected into 293T cells. Luciferase activity in lysates was measured $48 \mathrm{~h}$ after transfection and expressed as fold activity over the empty vector. (C) Sp1 and Sp3 binding to the COMMD1 promoter at $-0.1 \mathrm{~kb}$ was assessed by a chromatin immunoprecipitation assay using nuclear extracts of 293 T cells. Values are expressed as the mean \pm standard error from three independent experiments. P-values were determined by analysis of variance followed by Dunnett's test. ${ }^{* * *} \mathrm{P}<0.001$ vs. $-0.25 \mathrm{~kb}$ or the control. ns, not significant; Luc, luciferase; COMMD1, copper metabolism Murrl domain containing 1 ; con, control; Sp, specificity protein; mt, mutant.

opment in mice study. When we performed mildly knockdown Sp1, COMMD1 expression decreased (Fig. 3E and F). Sp1 is important transcription factor for COMMD1 transcription.

Although Sp3 clearly suppressed COMMD1 promoter activity, Sp3 overexpression did not suppress the mRNA expression of COMMD1. COMMD1 protein expression was upregulated by $\mathrm{Sp} 3$ overexpression; however, $\mathrm{Sp} 3$ overexpression did not increase COMMD1 mRNA levels and did not regulate COMMD1 transcription in the presence of Sp1. Sp1 and $\mathrm{Sp} 3$ expression levels are important for the transcription of certain genes $(26,27)$. While Sp3 decreased COMMD1 expression in Sp1-knockdown cells, the transcriptional regulation of the COMMD1 gene by Sp1 and Sp3 has remained to be fully elucidated. However, it is apparent that $\mathrm{Sp1}$ upregulates COMMD1 transcription and expression. By contrast, Sp3 may be involved in COMMD1 transcription and expression but its effect is dependent on the presence of Sp1.

A mutagenesis analysis demonstrated that the mutation in the first Sp1 site (mt1) suppressed promoter activity, while the mutation in the second site (mt2) enhanced it. These results suggested that the first site was the activator site of Sp1 binding and the second site was the inhibitory site. Previous studies reported that $\mathrm{Sp} 1$ acts as an activator and repressor in the same promoter region $(28,29)$. The present study investigated different mutant sequences in the second Sp1-binding site (mt2-2) by mutation analysis and identified that they also enhanced COMMD1 promoter activity. Although each of the two sites regulated COMMD1 promoter activity in the reporter assay, the transcription of endogenous COMMD1 by Sp1 may be regulated in the most closed Sp1-binding site.

The transcription factor $\mathrm{Sp1}$ upregulates the promoter activity and expression of COMMD1, while Sp3 suppresses the promoter activity at the steady state. Therefore, the present study indicated that $\mathrm{Sp} 1$ and $\mathrm{Sp} 3$ regulate the basal transcription and expression of COMMD1. However, a previous study by our group indicated that interferon- $\alpha$ (IFN $\alpha)$ and the Toll-like receptor 7/8 agonist R848 induced COMMD1 mRNA in a promonocyte cell line (10). In pathogen infection, particularly with viruses, IFN $\alpha$ produced in response to infection may regulate COMMD1 transcription. The present in silico analysis revealed the presence of several putative transcription factor-binding sites for STAT and interferon regulatory factor in the COMMD1 promoter region. However, in the present study, NF- $\mathrm{KB}$ and JAK inhibitor treatment did not suppress COMMD1 promoter activity in 293T cells. In several cancers and IBD, COMMD1 expression has been reported to 
be suppressed $(8,21)$, suggesting that inflammation-associated proteins regulate COMMD1 transcription. Further investigation of the transcriptional regulation of COMMD1 under these conditions or in specific cell types is required. COMMD1 is a protein associated with multiple cellular pathways, including copper homeostasis and NF-кB and hypoxia-inducible factor-1 signaling. In the present study, Sp1 was identified as a transcriptional regulator of COMMD1. These results demonstrated the molecular mechanisms of the regulation of COMMD1 gene expression by $\mathrm{Sp} 1$ at the steady state.

\section{Acknowledgements}

The present study was supported by the Research Program on HIV/AIDS (grant no. 17fk0410208h002 to S.O. and E.K.) of the Japan Agency for Medical Research and Development and a Grant-in-Aid for Research Activity Start-up (grant no. $16 \mathrm{H} 07080$ to E.K.) from the Ministry of Education, Science, Sports and Culture of Japan. The authors would like to thank Ms. Y. Endo and Ms. Y. Kanagawa for their secretarial assistance and Ms. I. Suzu and Ms. S. Fujikawa (Center for AIDS Research, Kumamoto University, Kumamoto, Japan) for their research assistance.

\section{Competing interests}

The authors declare that they have no competing interests.

\section{References}

1. Burstein E, Hoberg JE, Wilkinson AS, Rumble JM, Csomos RA, Komarck CM, Maine GN, Wilkinson JC, Mayo MW and Duckett CS: COMMD proteins, a novel family of structural and functional homologs of MURR1. J Biol Chem 280: 22222-22232, 2005.

2. Tao TY, Liu F, Klomp L, Wijmenga C and Gitlin JD: The copper toxicosis gene product Murr1 directly interacts with the Wilson disease protein. J Biol Chem 278: 41593-41596, 2003.

3. de Bie P, van de Sluis B, Burstein E, van de Berghe PV, Muller P, Berger R, Gitlin JD, Wijmenga C and Klomp LW: Distinct Wilson's disease mutations in ATP7B are associated with enhanced binding to COMMD1 and reduced stability of ATP7B Gastroenterology 133: 1316-1326, 2007.

4. Vonk WI, de Bie P, Wichers CG, van den Berghe PV, van der Plaats R, Berger R, Wijmenga C, Klomp LW and van de Sluis B: The copper-transporting capacity of ATP7A mutants associated with Menkes disease is ameliorated by COMMD1 as a result of improved protein expression. Cell Mol Life Sci 69: 149-163, 2012 .

5. Ke Y, Butt AG, Swart M, Liu YF and McDonald FJ: COMMD1 downregulates the epithelial sodium channel through Nedd4-2. Am J Physiol Renal Physiol 298: F1445-F1456, 2010.

6. Maine GN, Mao X, Komarck CM and Burstein E: COMMD1 promotes the ubiquitination of NF-kappaB subunits through a cullin-containing ubiquitin ligase. EMBO J 26: 436-447, 2007.

7. Drevillon L, Tanguy G, Hinzpeter A, Arous N, de Becdelièvre A, Aissat A, Tarze A, Goossens M and Fanen P: COMMD1-mediated ubiquitination regulates CFTR trafficking. PLoS One 6: e18334, 2011.

8. van de Sluis B, Mao X, Zhai Y, Groot AJ, Vermeulen JF, van der Wall E, van Diest PJ, Hofker MH, Wijmenga C, Klomp LW, et al: COMMD1 disrupts HIF-1alpha/beta dimerization and inhibits human tumor cell invasion. J Clin Invest 120: 2119-2130, 2010

9. Ganesh L, Burstein E, Guha-Nijogi A, Louder MK, Mascola JR, Klomp LW, Wijmenga C, Duckett CS and Nabel GJ: The gene product Murrl restricts HIV-1 replication in resting $\mathrm{CD}^{+}$ lymphocytes. Nature 426: 853-857, 2003.
10. Taura M, Kudo E, Kariya R, Goto H, Matsuda K, Hattori S, Vaeteewoottacharn K, McDonald F, Suico MA, Shuto T, et al: COMMD1/Murr1 reinforces HIV-1 latent infection through IкB- $\alpha$ stabilization. J Virol 89: 2643-2658, 2015.

11. Beishline K and Azizkhan-Clifford J: Sp1 and the 'hallmarks of cancer'. FEBS J 282: 224-258, 2015.

12. Resendes KK and Rosmarin AG: Sp1 control of gene expression in myeloid cells. Crit Rev Eukaryot Gene Expr 14: 171-181, 2004.

13. Li L, He S, Sun J and Davie J: Gene regulation by Sp1 and Sp3. Biochem Cell Biol 82: 460-471, 2004.

14. Majello B, De Luca P and Lania L: Sp3 is a bifunctional transcription regulator with modular independent activation and repression domains. J Biol Chem 272: 4021-4026, 1997.

15. Taura M, Kariya R, Kudo E, Goto H, Iwawaki T, Amano M, Suico MA, Kai H, Mitsuya H and Okada S: Comparative analysis of ER stress response into HIV protease inhibitors: Lopinavir but not darunavir induces potent ER stress response via ROS/JNK pathway. Free Radic Biol Med 65: 778-788, 2013.

16. Suico MA, Taura M, Kudo E, Gotoh K, Shuto T, Okada S and Kai H: The ETS factor myeloid Elf-1-like factor (MEF)/Elf4 is transcriptionally and functionally activated by hypoxia. Biol Pharm Bull 39: 641-647, 2016.

17. Kudo E, Taura M, Matsuda K, Shimamoto M, Kariya R, Goto H, Hattori S, Kimura S and Okada S: Inhibition of HIV-1 replication by a tricyclic coumarin GUT-70 in acutely and chronically infected cells. Bioorg Med Chem Lett 23: 606-609, 2013.

18. Taura M, Eguma A, Suico MA, Shuto T, Koga T, Komatsu K, Komune T, Sato T, Saya H, Li JD and Kai H: p53 regulates Toll-like receptor 3 expression and function in human epithelial cell lines. Mol Cell Biol 28: 6557-6567, 2008.

19. Schmittgen TD and Livak KJ: Analyzing real-time PCR data by the comparative C(T) method. Nat Protoc 3: 1101-1108, 2008.

20. Greenwel P, Inagaki $\mathrm{Y}, \mathrm{Hu} \mathrm{W}$, Walsh $\mathrm{M}$ and Ramirez F: Sp1 is required for the early response of alpha2(I) collagen to transforming growth factor-beta1. J Biol Chem 272: 19738-19745, 1997.

21. Li H, Chan L, Bartuzi P, Melton SD, Weber A, Ben-Shlomo S, Varol C, Raetz M, Mao X, Starokadomskyy P, et al: Copper metabolism domain-containing 1 represses genes that promote inflammation and protects mice from colitis and colitis-associated cancer. Gastroenterology 147: 184-195.e3, 2014.

22. Yeh DW, Chen YS, Lai CY, Liu YL, Lu CH, Lo JF, Chen L, Hsu LC, Luo Y, Xiang R and Chuang TH: Downregulation of COMMD1 by miR-205 promotes a positive feedback loop for amplifying inflammatory- and stemness-associated properties of cancer cells. Cell Death Differ 23: 841-852, 2016.

23. O'Connor L, Gilmour J and Bonifer C: The role of the ubiquitously expressed transcription factor $\mathrm{Sp} 1$ in tissue-specific transcriptional regulation and in disease. Yale J Biol Med 89: $513-525,2016$.

24. Zhang DE, Hetherington CJ, Tan S, Dziennis SE, Gonzalez DA, Chen HM and Tenen DG: Sp1 is a critical factor for the monocytic specific expression of human CD14. J Biol Chem 269: 11425-11434, 1994

25. Tone M, Powell MJ, Tone Y, Thompson SA and Waldmann H: IL-10 gene expression is controlled by the transcription factors Sp1 and Sp3. J Immunol 165: 286-291, 2000.

26. Le Goff W, Guerin M, Petit L, Chapman MJ and Thillet J: Regulation of human CETP gene expression: Role of SP1 and SP3 transcription factors at promoter sites-690, -629 , and -37 . J Lipid Res 44: 1322-1331, 2003.

27. Apt D, Watts RM, Suske G and Bernard HU: High Sp1/Sp3 ratios in epithelial cells during epithelial differentiation and cellular transformation correlate with the activation of the HPV-16 promoter. Virology 224: 281-291, 1996.

28. Encarnacao PC, Ramirez VP, Zhang C and Aneskievich BJ: $\mathrm{Sp}$ sites contribute to basal and inducible expression of the human TNIP1 (TNFo-inducible protein 3-interacting protein 1) promoter. Biochem J 452: 519-529, 2013.

29. Li R, Hodny Z, Luciakova K, Barath P and Nelson BD: Sp1 activates and inhibits transcription from separate elements in the proximal promoter of the human adenine nucleotide translocase 2 (ANT2) gene. J Biol Chem 271: 18925-18930, 1996. 